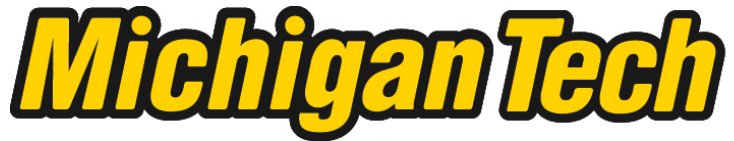 \\ Michigan Technological University Create the Future Digital Commons @ Michigan Tech
}

Dissertations, Master's Theses and Master's Reports - Open

Dissertations, Master's Theses and Master's

Reports

2015

STUDY OF NON-RECIPROCAL DICHROISM IN PHOTONIC STRUCTURES

Anindya Majumdar

Michigan Technological University

Follow this and additional works at: https://digitalcommons.mtu.edu/etds

Part of the Physics Commons

Copyright 2015 Anindya Majumdar

\section{Recommended Citation}

Majumdar, Anindya, "STUDY OF NON-RECIPROCAL DICHROISM IN PHOTONIC STRUCTURES", Master's report, Michigan Technological University, 2015.

https://doi.org/10.37099/mtu.dc.etds/970

Follow this and additional works at: https://digitalcommons.mtu.edu/etds

Part of the Physics Commons 


\title{
STUDY OF NON-RECIPROCAL DICHROISM IN PHOTONIC STRUCTURES
}

\author{
By \\ Anindya Majumdar
}

\begin{abstract}
A REPORT
Submitted in partial fulfillment of the requirements for the degree of MASTER OF SCIENCE in Physics
\end{abstract}

\section{MICHIGAN TECHNOLOGICAL UNIVERSITY}

2015

(C) 2015 Anindya Majumdar 
This report has been approved in partial fulfillment of the requirements for the degree of MASTER OF SCIENCE in Physics.

Department of Physics

Report Advisor: Dr. Miguel Levy

Committee Member: Dr. Ramy El-Ganainy

Committee Member: Dr. Sean Kirkpatrick

Department Chair: Dr. Ravindra Pandey 


\section{$\underline{\text { Table of Contents }}$}

Acknowledgement i

Abstract

$\begin{array}{ll}\text { Abbreviations } & \text { ii }\end{array}$

I. NON-RECIPROCAL DICHROISM 1

I.1 Introduction 1

I.2 Formulation 1

I.3 Our Studies and Results 3

I.4 Conclusions 5

II. FROZEN MODES

$\begin{array}{ll}\text { II.1 Motivation } & 7\end{array}$

$\begin{array}{ll}\text { II.2 Theory } & 7\end{array}$

II.3 Our Studies and Results 9

SUMMARY AND FUTURE PROSPECTS 12

REFERENCES 13

APPENDIX 


\section{Acknowledgement}

I wholeheartedly thank my advisor Dr. Levy for providing his valuable guidance to me during the course of my studies in Physics. This work could not have been done without his valuable and timely inputs. He has been consistently available to discuss any problems I might have faced during the course of these studies.

I am also thankful to my family for their love and support throughout my career. I would like to thank Dr. El-Ganainy and Dr. Kirkpatrick for being my committee members. I also thank Ms. Divya Kamath, who is a PhD candidate in Environmental Engineering at MTU, for her immense help in efficiently writing this report. Finally, I thank the entire Michigan Tech community, and the Department of Physics in particular, for their timely assistance regarding various activities over the last two years. 


\begin{abstract}
Non-reciprocal phenomena are widely used in photonic devices. Important applications such as isolators and circulator waveguide structures depend on non-reciprocal effects. The basis of these phenomena is a difference in phase or refractive index for an electromagnetic wave of a given polarization as it travels through a medium in opposite directions. Generally this applies to the real part of the refractive index. The present report addresses a less studied phenomenon in non-reciprocal propagation, namely differences in optical absorption loss for a given polarization state in opposite propagation directions, a phenomenon we have termed non-reciprocal dichroism. Nonreciprocal dichroism can be defined as a difference in absorption between two opposite directions of wave traversal. In this report, we attempt to study the non-reciprocal dichroism in transversely magnetized optical waveguides. Further, we investigate the existence of frozen modes in waveguide structures. Such modes can give rise to conditions wherein a wave has neither velocity nor acceleration, and thus can be thought of as "frozen" in a medium. In the presence of absorption loss, this leads to large absorption levels and can be considered as an extreme case of non-reciprocal dichroism.
\end{abstract}

\title{
Abbreviations
}

TE : Transverse - Electric

TM : Transverse- Magnetic 


\section{I.1 Introduction}

\section{NON-RECIPROCAL DICHROISM}

TE-TM mode-index matching had once been the primary principle in use for Faraday rotation-based devices. However, application of non-reciprocal phase shift effect to on-chip isolator development has been actively pursued as a promising alternative over the last two decades [1-15]. This relaxes the necessity of stringent control of waveguide parameters [16-18] that made Faraday-rotation-based devices very difficult and costly to fabricate. A signature feature of this effect is the difference in TM (or TE) mode indices between oppositely propagating beams in transversely magnetized waveguide geometries. The coupling of longitudinal and transverse optical electric field components of the propagating wave results in a dispersion relation that is asymmetric upon reversal in propagation, leading to nonreciprocal propagation.

This effect has been applied for various integrated-device realizations, including nonreciprocal Mach-Zehnder interferometers with ferrite cores [48], or with ferrite cover-layers in silicon-on-insulator platforms [9-12], ringresonators [13], and nonreciprocal mode conversion devices [19]. No analysis of nonreciprocal propagation loss, or of intrinsic nonreciprocal absorption in these transversely magnetized geometries were found by us. It should be indeed expected that in the presence of absorption, differences in interaction of the optical beam with the material will give rise to differences in propagation constant, and thus to non-reciprocity in the losses. Thus, nonreciprocal dichroism, which is a difference in the intrinsic absorption for a given polarization upon reversal in propagation direction, should be expected.

\section{I.2 Formulation}

We consider a transversely magnetized optical waveguide, with in-plane magnetization along the y-direction and wave propagation along the z-axis. 


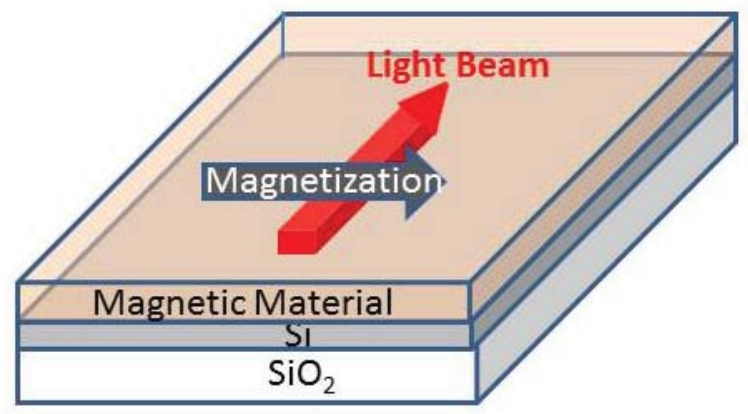

Fig. 1. Geometry of the slab waveguide structure under consideration The dielectric permittivity is then given by

$$
\varepsilon=\left[\begin{array}{ccc}
\varepsilon_{x x} & 0 & \varepsilon_{x z}=i g \\
0 & \varepsilon_{y y} & 0 \\
\varepsilon_{z x}=-i g & 0 & \varepsilon_{z z}
\end{array}\right]
$$

Here $g$ is referred to as the gyrotropy parameter. In the absence of absorption loss, the parameters $\varepsilon_{i i}, i=x, y, z$, and $g$, are all real.

For an isotropic garnet material, $\varepsilon_{x x}=\varepsilon_{y y}=\varepsilon_{z z}$. The corresponding dispersion relation for a slab waveguide under these conditions is given by,

$$
\begin{gathered}
d\left(k_{0}^{2} \epsilon_{f}-\beta^{2}\right)^{1 / 2} \\
=\tan ^{-1}\left\{\frac{\epsilon_{f}}{\left(k_{0}^{2} \epsilon_{f}-\beta^{2}\right)^{1 / 2}} \times\left[\frac{\left(\beta^{2}-k_{0}^{2} \epsilon_{e f f}\right)^{1 / 2}}{\epsilon_{e f f}}+\frac{\beta \epsilon_{x z}}{\epsilon_{x x} \epsilon_{e f f}}\right]\right\} \\
+\tan ^{-1}\left\{\frac{\epsilon_{f}}{\left(k_{0}^{2} \epsilon_{f}-\beta^{2}\right)^{1 / 2}}\left[\frac{\left(\beta^{2}-k_{0}^{2} \epsilon_{s}\right)^{1 / 2}}{\epsilon_{S}}\right]\right\}
\end{gathered}
$$

where $\epsilon_{e f f}=\epsilon_{x x}-g^{2} / \epsilon_{x x}, k_{0}=2 \pi / \lambda$ is the vacuum wave number, $\beta$ is the modal propagation constant and $d$ and $\epsilon_{f}$ are the thickness and dielectric constant of the waveguide film, respectively [10]. The derivation of the dispersion relation is in the appendix.

Upon introducing the effect of absorption in the magnetic cover layer, the diagonal elements of the dielectric tensor acquire an imaginary part. Thus, we have $\epsilon_{x x}=\epsilon^{\prime}+i \epsilon^{\prime \prime}$ for real $\epsilon^{\prime}$ and $\epsilon^{\prime \prime}$. These real and imaginary parts of the dielectric tensor are related to the refractive index $n$ and the extinction coefficient $k$ as: 


$$
\epsilon^{\prime}=n^{2}-k^{2} \text { and } \epsilon^{\prime \prime}=2 n k
$$

\section{I.3 Our Studies and Results}

For an actual guiding material, a certain amount of loss is always present, which affects the device performance. This necessitates an understanding of the effect of non-reciprocity. The effect may also be explored for the possibilities of technological uses of controlled nonreciprocal absorption losses. Thus, we compute the nonreciprocal difference in absorption loss for waveguides with cover layers under various extinction coefficients; these include rare-earth-substituted iron garnets, such as cerium-substituted yttrium iron garnets, as well as garnets having enhanced absorptivity similar to those of high-loss cobalt-substituted and other iron garnets on silicon-on-insulator platforms $[9-12,21,22]$.

We explore the consequences of the dispersion relation for the case of a transversely-magnetized slab waveguide consisting of a silicon-on-insulator slab with a magnetic-garnet cover layer (Fig. 1), for the case of TM polarization. A free-space optical wavelength of $1.55 \mu \mathrm{m}$ is assumed. It is to be noted that we neglect the effect of absorption loss in the silicon film and silicon-dioxide substrate, and consider the contribution of material absorption on the gyrotropy parameter $g$ to be negligible, i.e., that $g$ remains purely real and that its magnitude is not affected by the optical absorption in the material. In general, circular dichroism will affect the gyrotropy as articulated through the Kramers-Kronig relations, but this effect is not included in the present analysis.

We note that the phase of the electric field component for a forward propagating wave is given by $\beta z-\omega t$. When the propagation constant is complex $\left(\beta=\beta_{r}+i \beta_{i}\right)$ as in our study, this phase-bearing factor gets modified to $e^{i\left(\beta_{r} z-\omega t\right)} e^{-\beta_{i} z}$. We calculate the nonreciprocal phase-shift, $\Delta \beta=\beta_{f w}-$ $\beta_{b w}$, for a range of thicknesses of the silicon layer from $210 \mathrm{~nm}$ to $600 \mathrm{~nm}$, where $\beta_{f w}$ and $\beta_{b w}$ are the forward and backward propagation constants, respectively. 
The cover-layer refractive index used here is 2.22, typical of magnetic garnets, and the response of the system for $k$ ranging between 0.0003 and 0.03 is investigated. We use a value for $\left|\varepsilon_{x z}\right|=g$ of 0.0086 throughout, corresponding to that of cerium-substituted yttrium iron garnet (Ce:YIG) with one cerium ion per-formula-unit grown on (111)-oriented cation ( $\mathrm{Ca}, \mathrm{Mg}, \mathrm{Zr}$ )-doped Gd3Ga5O12 (NOG) substrate [9]. For simplicity, this gyrotropy is assumed to remain the same for all values of the extinction coefficient considered here.

Typical values of the extinction coefficient $k$ for pulsed-laser-deposited Ce:YIG on silicon are approximately 0.0003 [13]. Similarly, those of liquidphase-epitaxially-grown bismuth-substituted yttrium iron garnets (Bi:YIG), at one bismuth-ion per formula unit and $1.55 \mu \mathrm{m}$ wavelength hover around 0.0002 [21]. Cobalt-substituted iron garnets have exhibited much larger intrinsic optical loss in the $1.55 \mu \mathrm{m}$ wavelength range as well as large gyrotropies [22]. Extinction coefficients on the order of $k=0.012$, corresponding to $\mathrm{Co}^{2+}$ ion substitutions into the tetravalent iron sites in the garnet have been reported [22]. Precision doping with compensating dopants such as $\mathrm{Ca}^{2+}$ and $\mathrm{Mg}^{2+}$ have been used to achieve perfect stoichiometry in liquid-phase-epitaxial bismuth-substituted iron garnets, also yielding significant intrinsic absorption with values of $k$ as large as 0.001 at $1.15 \mu \mathrm{m}$ wavelengths [23]. Thus there exists a variety of material systems encompassing a wide range of realistic extinction coefficient. We also note that there are magnetic materials possessing much higher extinction coefficients, such as for example transitions metals are also available. An iron cover, at $1.55 \mu m$ exhibits values of $g \sim 0.56$ and $k \sim 5.53$.
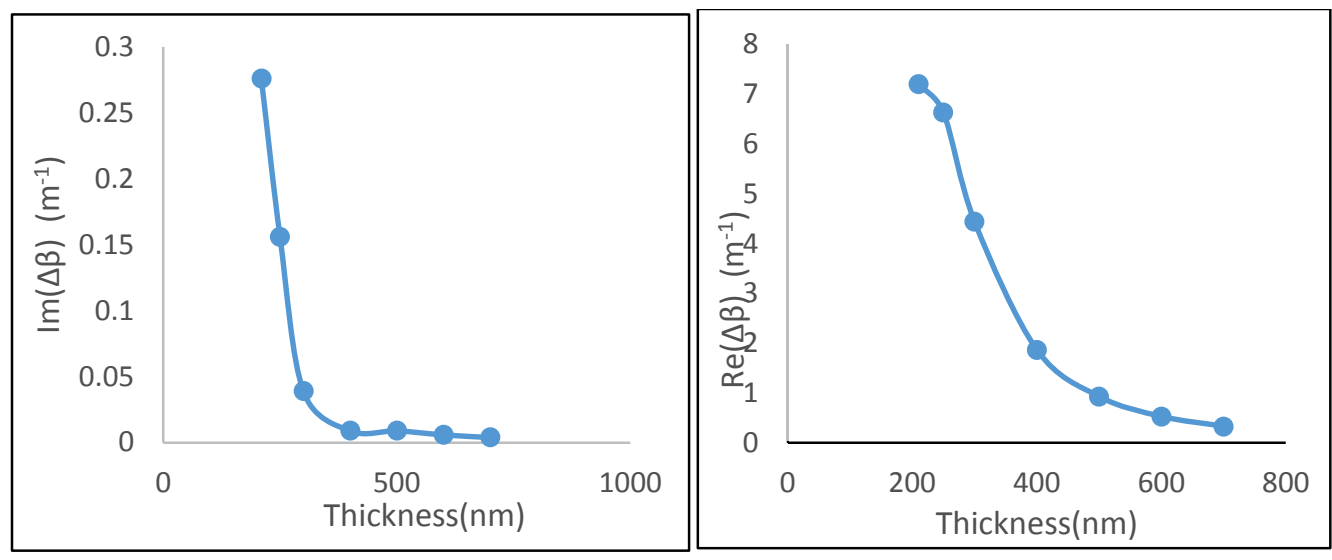

Fig. 2. Calculated variation in real and imaginary parts of $\Delta \beta$ with $\mathrm{k}=0.03$ 
Figure 2 shows the thickness dependence of the real and the imaginary parts of $\Delta \beta$ for $\mathrm{k}=0.03$. As the thickness increases beyond $210 \mathrm{~nm}$, both the real and imaginary parts decrease considerably. This decrease is quite steep between $210 \mathrm{~nm}$ and $400 \mathrm{~nm}$. It tends to be more gradual for the real part of the nonreciprocal phase shift as the thickness increases beyond $400 \mathrm{~nm}$. The nonreciprocity in dichroism appears to level off between $400 \mathrm{~nm}$ and $600 \mathrm{~nm}$, showing a slight increase around $500 \mathrm{~nm}$.

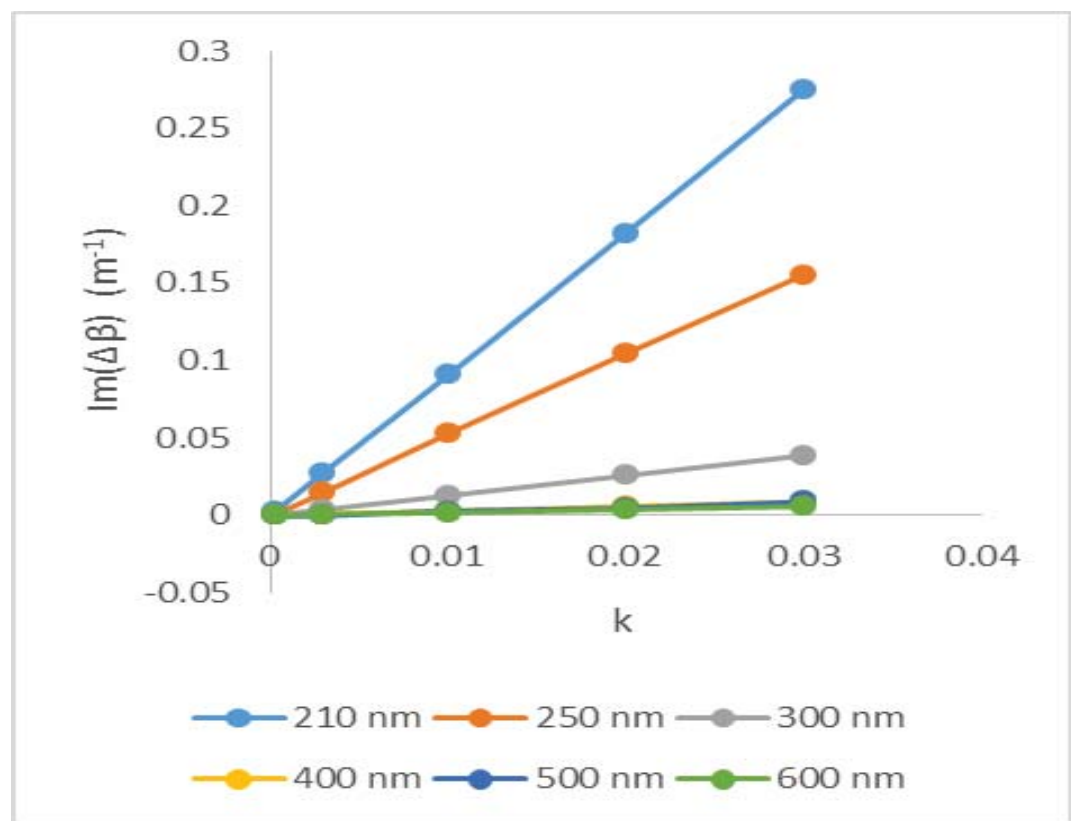

Fig. 3. Variation in the imaginary part of $\Delta \beta$ with $\mathrm{k}$ for different film thicknesses

We also observe, as shown in Fig. 3, that the imaginary part of the nonreciprocal phase shift increases linearly with the value of the propagation constant, for each different cover thickness. The change is steeper at lower thicknesses, where the loss is more pronounced. As the intrinsic extinction coefficient of the magnetic material increases the loss difference between forward and backward propagation also increases.

\section{I.4 Conclusions}

It is clear from the analysis presented above that significant differences in optical absorption in opposite propagation directions can be realized under 
certain conditions in magneto-optic media. This understanding opens up the possibility for new types of non-reciprocal devices relying on differential absorption rather than destructive interference or the use of polarizers as in previous architectures. Notice also that if the optical wave could be slowed down significantly in one of the propagation directions this would lead to extreme levels of differential absorptions or non-reciprocal dichroism. This question is addressed in the following section. 


\section{FROZEN MODES}

\section{II.1 Motivation}

Unidirectional magneto-photonic-crystal layered structures able to generate a frozen electromagnetic mode in one propagation direction were proposed by A.Figotin and I.Vitebskiy in 2003 [20]. Recently, frozen-mode magneto-optic layered structures have also been proposed for unidirectional lasing applications [25]. In these, the differences in the extent of the interaction of the optical beam with the underlying material system play an even more pronounced role than the optical isolator prototypes mentioned in the introduction to non-reciprocal dichroism. Extreme cases of non-reciprocal dichroism can be obtained by extending the application of these structures to on-chip optical waveguides in the presence of absorption loss or gain. These would open up the possibility for complete unidirectional elimination of transmitted optical power or mirror-less unidirectional lasing in waveguide geometries at optical frequencies.

\section{II.2 Theory}

As mentioned, frozen modes in transversely magnetized optical waveguide structures provide an extreme case for the implementation of nonreciprocal dichroism. Completely stopping the propagation of an optical beam in one direction in the presence of absorption loss constitutes a limiting case of the difference in the extent of the interaction of the beam with the material constituents of the waveguide channel in opposite directions. This can be realized in a magneto-photonic crystal. Theoretically, the complete nonreciprocal elimination of the optical power is feasible in that case.

The existence of a frozen mode is related to the presence of an inflection point in one of the spectral branches of the photonic band structure, with $\frac{\partial \omega}{\partial k}=0, \frac{\partial^{2} \omega}{\partial k^{2}}=0, \frac{\partial^{3} \omega}{\partial k^{3}} \neq 0$ at some $k=k_{0}$ under the condition that $\omega(k) \neq \omega(-k)[20]$. Figotin and Vitebskiy discuss the photonic crystal symmetry conditions necessary for the existence of such an inflection point, 
and propose layered stack structures satisfying these symmetry conditions [20].

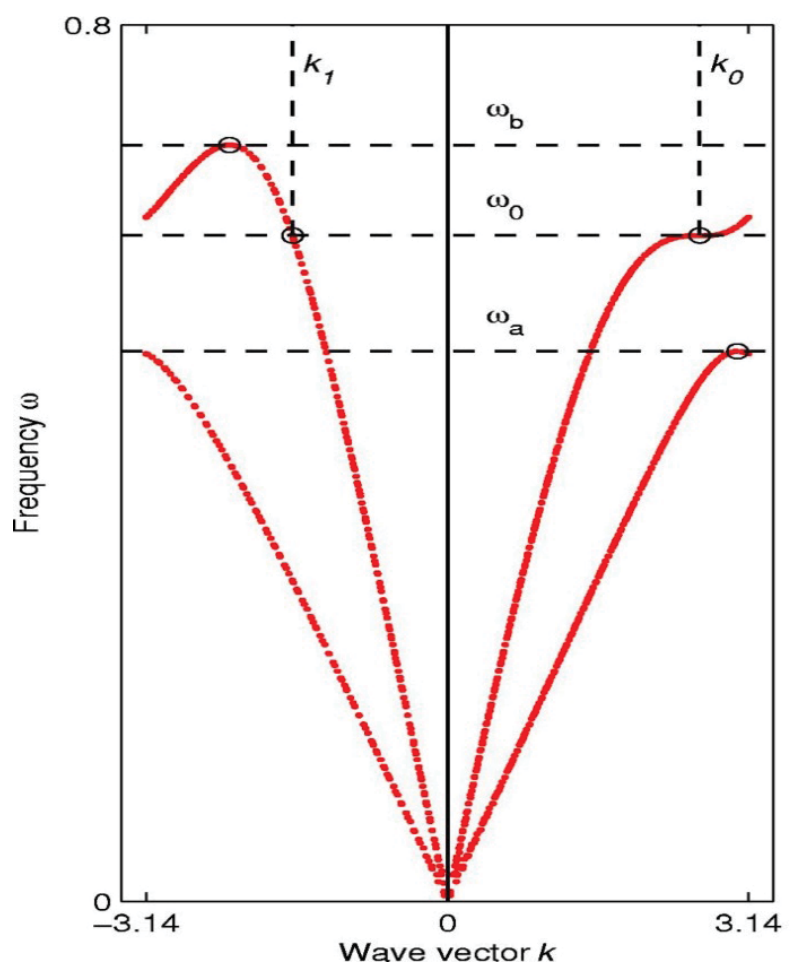

Fig. 4. Displaying unidirectionality at $\omega=\omega_{0}$ in a band structure

(Source: Figotin and Vitebskiy, 2003 [20])

A necessary condition for asymmetric band structures is that the symmetry operations of the photonic structure do not transform $\boldsymbol{k}$ into $\boldsymbol{- k}$ [24]. The structure shown in Fig. 5 is an example of a magneto-photonic crystal waveguide structure that can be used as a basis to study the implementation of the extreme type of nonreciprocal dichroism discussed above. This structure has just one symmetry transformation: a 2-fold rotation about the $\mathrm{x}$ axis followed by time reversal. This transformation takes $\boldsymbol{k}$ into $\boldsymbol{k}$, so it satisfies the condition that the symmetry operations of the photonic structure do not transform $\boldsymbol{k}$ into $\boldsymbol{k} \boldsymbol{k}$.

In addition, the slanted configuration of holes forces all the modes to be degenerate and mix together to build a stationary mode in one propagation direction. This will not happen if, for example, all the holes lined up along the y-direction. In that case the magnetic symmetry of the structure would also acquire mirror planes $m_{y}$ and $m_{z}^{\prime}$. Neither of them changes $k_{z}$ to $-k_{z}$, hence, the spectral asymmetry will persist, but $\mathrm{m}_{\mathrm{y}}$ will result in separation of TE and 
TM polarizations and remove the degeneracy needed to build up stationary modes. It should be pointed out that the waveguide should not be single-mode but should be able to support four modes for this to be realized.

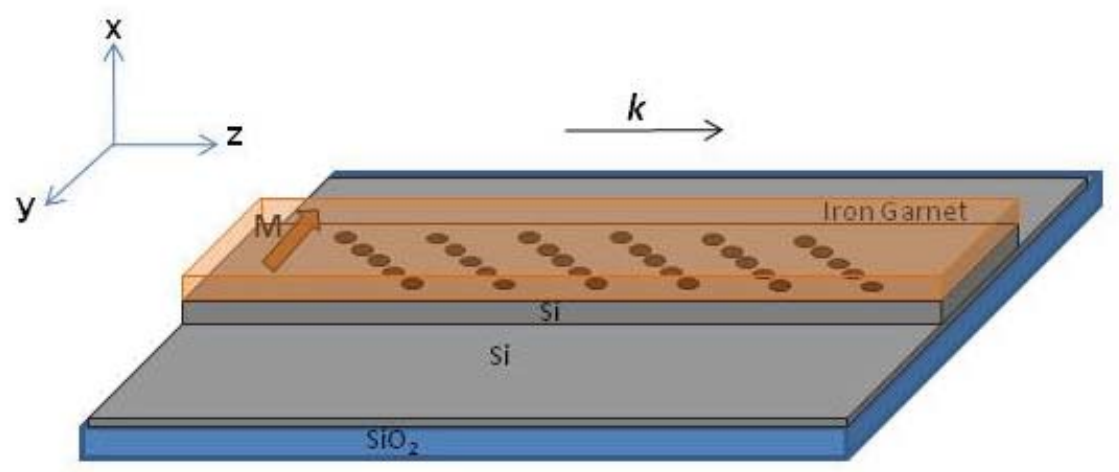

Fig. 5. A sample structure satisfying the geometrical conditions mentioned by Figotin and Vitebskiy

(Suggested by Dr. Miguel Levy)

\section{II.3 Our Studies and Results}

This part of our study is based on computation work performed on a commercial software "RSoft". First, we needed to verify whether the software was able to mimic results from known theory. For this, we took a slab waveguide to perform our calculations and obtained the following band structure 


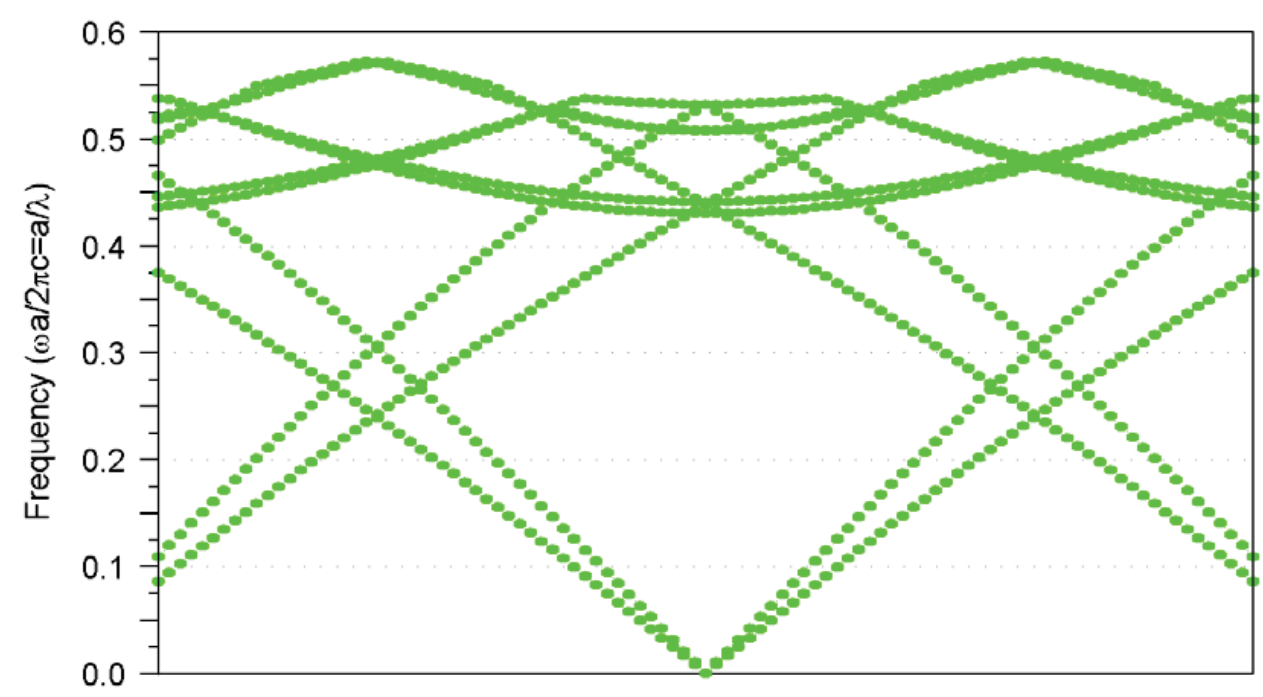

A

B

Fig.6. Band structure obtained for a slab waveguide, with A and B representing zone boundaries

The parameter ' $a$ ' is the period of the structure needed to be set into the software while creating the structure, such that normalized frequency axis has values in terms of this period. For all the studies in this report, the value of ' $a$ ' was set as $1.5 \mu \mathrm{m}$. The points ' $\mathrm{A}$ ' and ' $\mathrm{B}$ ' were Brillouin zone boundaries, also set into the software after calculating using the lattice properties. We observed that the band-bending points for the two lowest bands, falling within the window of theoretical calculations. Also, no bandgaps were observed from the slab waveguide. These comparisons took us to the conclusion that the results from our RSoft calculations mimicked the expectations from theory [26].

Next, we performed similar calculation on a structure with periodic holes, placed $1.5 \mu \mathrm{m}$ apart. The structure had a refractive index of 1.5. The obtained band structure is shown in Fig.7. The definitions of all parameters remain the same. We observe a bandgap centered at $\frac{a}{\lambda}=0.34$. This value is within $3 \%$ of the theoretical value expected from Bragg reflection calculations. The small difference can be postulated to arise from the fact that the effective refractive index of the structure is slightly different from the actual refractive index of the material. 


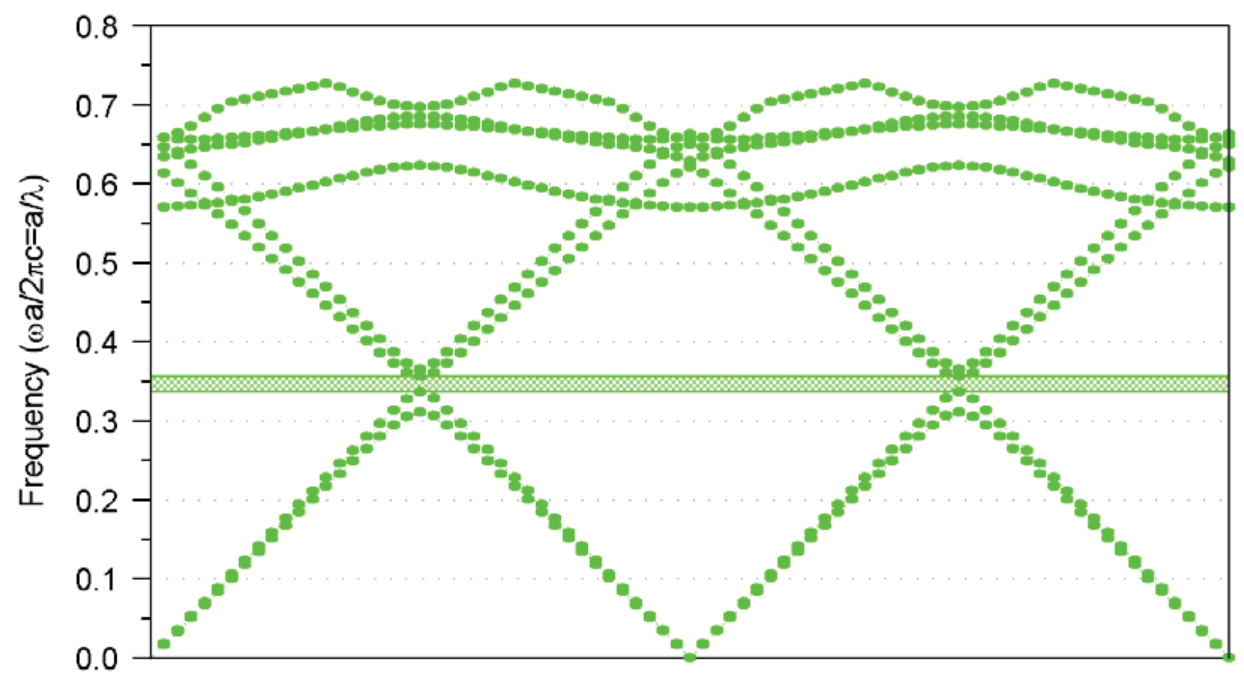

A

B

Fig.7. Band structure of a linear periodic material with holes

The agreement between theoretical calculations and results obtained from software motivated us to look for band structures of other structures using this tool, in the search of frozen modes. For example, Fig.8 represents the band structure of a pattern
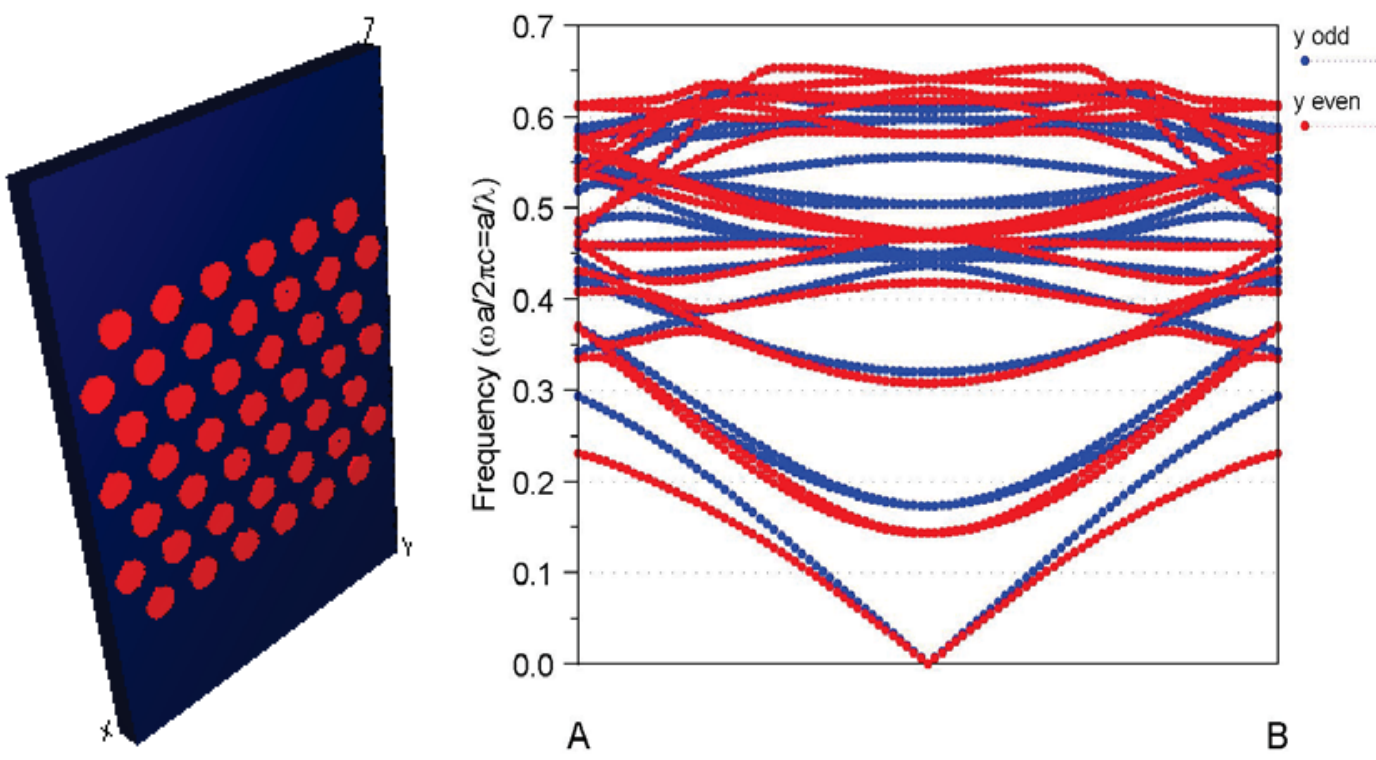

Fig.8. A hexagonal pattern of holes in a slab and its band structure

of holes periodically placed in a hexagonal format, in a slab of refractive index 1.5. As can be seen from the figure, some of the higher modes tend to flatten 
out near the zone boundaries- what we can call a "shoulder-like" pattern. Similar patterns were found in some other configurations as well. The future studies in this direction lie in the prospect of searching for more prominent such patterns, and more importantly, being able to design structures wherein we can control this flattening out. Using magneto-optic effects in the design is an area that can be looked into for such patterns, which might lead to demonstrable dichroism.

\section{SUMMARY AND FUTURE PROSPECTS}

As mentioned, as per the best of our knowledge, no previous work has been done to examine absorption losses in calculations of the non-reciprocal phase shifts. On including the absorption effects, we have been able to calculate nonreciprocal phase shifts as well as difference in absorption losses in the forward and backward propagation of electromagnetic waves. A detailed derivation of the dispersion relation is attached as an appendix. We have also initiated an examination and design of various photonic crystal structures for the implementation of frozen modes. However, more detailed analysis is required before any such achievement. The fact that RSoft is able to reproduce results obtained from well-established theories indicates a highly promising direction in which this research can progress. 


\section{REFERENCES}

1. F. Auracher, H. Witte, "A new design for an integrated optical isolator," Optics Comm. 13, 435-438 (1975).

2. Y. Okamura, T. Negami, S. Yamamoto, "Integrated optical isolator and circulator using nonreciprocal phase shifters: a proposal," Appl. Optics 23, 1886-1889 (1984).

3. H. Dötsch, P. Hertel, B. Lührmann, S. Sure, H.P. Winkler, and M. Ye, "Applications of magnetic garnet films in integrated optics," IEEE Trans. on Magnetics 28, 2979-2984 (1992).

4. T. Shintaku, "Integrated optical isolator based on nonreciprocal higherorder mode conversion," Appl. Phys. Lett. 66, 2789-2791 (1995).

5. N. Bahlmann, M. Lohmeyer, O. Zhuromsky, H. Dötsch, P. Hertel, "Nonreciprocal coupled waveguides for integrated optical isolators and circulators for TM-modes," Optics Comm. 161, 330-337 (1999).

6. J. Fujita, M. Levy, R. M. Osgood, Jr., L. Wilkens, and H. Dötsch, "Waveguide Optical Isolator Based on Mach-Zehnder Interferometer," Appl. Phys. Lett. 76, 2158-2160 (2000).

7. J. Fujita, M. Levy, R. M. Osgood, Jr., L. Wilkens, and H. Doetsch, "Polarization-Independent Waveguide Optical Isolator based on Nonreciprocal Phase Shift," IEEE Photonics Technol. Lett. 12, 15101512 (2000).

8. M. Levy, "The On-Chip Integration of Magneto-Optic Waveguide Isolators," invited paper IEEE J. of Selected Topics in Quantum Electronics 8, No. 6, 1300 - 1306 (2002).

9. H. Yokoi, Y. Shoji and T. Mitsumoto, "Calculation of Nonreciprocal Phase Shift in Magnetooptic Waveguide with Si Guiding Layer," Japanese Journal of Applied Physics 43, 5871-5874 (2004).

10. R. L. Espinola, T. Izuhara, M.-C. Tsai, R. M. Osgood, Jr., and H. Dotsch, "Magneto-optical nonreciprocal phase shift in garnet/siliconon-insulator waveguides," Opt. Lett. 29, 941-943 (2004).

11. Y. Shoji, I. -W. Hsieh, R. M. Osgood, Jr., and T. Mizumoto, "Polarization-independent magneto-optical waveguide isolator using TM-mode nonreciprocal phase shift," J. Lightwave Technol. 25, 31083113 (2007).

12. Y. Shoji, T. Mizumoto, H. Yokoi, I-Wei Hsieh, and Richard M. Osgood, Jr., "Magneto-optical isolator with silicon waveguides fabricated by direct bonding," Appl. Phys. Lett. 92, 071117 (2008). 
13. L. Bi, J. Hu, P. Jiang, D. H. Kim, G. F. Dionne, L. C. Kimerling and C. A. Ross, "On-chip optical isolation in monolithically integrated nonreciprocal optical resonators," Nature Photonics 5, 758-762 (2011).

14. M.-C. Tien, T. Mizumoto, P. Pintus, H. Kromer, and J. E. Bowers, "Silicon ring isolators with bonded nonreciprocal magneto-optic garnets," Opt. Express 19, 11740-11745 (2011).

15. T. Mizumoto, R. Takei and Y. Shoji, "Waveguide optical isolators for integrated optics," IEEE Journal of Quantum Electronics 48, 252- 260 (2012).

16. R. Wolfe, R. A. Lieberman, V. J. Fratello, R. E. Scotti, and N. Kopylov, "Etch-tuned ridged waveguide magneto- optic isolator," Appl. Phys. Lett. 56, 426-428 (1990).

17. M. Levy, I. Ilic, R. Scarmozzino, R. M. Osgood, Jr., R. Wolfe, C. J. Gutierrez, and G. Prinz, "Thin-Film-Magnet Magneto-optic Waveguide Isolator," IEEE Photonics Technology Letters 5 198-200 (1993).

18. M. Levy, H. Hegde, F. J. Cadieu, R. Wolfe, V. J. Fratello, and R. M. Osgood, Jr., "Integrated Optical Isolators with Sputter-Deposited ThinFilm Magnets," IEEE Phot. Technol. Lett. 8, 903-905 (1996).

19. T. Shintaku, "Integrated optical isolator based on efficient nonreciprocal radiation mode conversion," Appl. Phys. Lett. 73, 19461948 (1998).

20. A. Figotin and I. Vitebskiy, "Electromagnetic unidirectionality in magnetic photonic crystals," Phys. Rev. B 67, 165210 (2003).

21. V. Doormann, J.P. Krumme, and H. Lenz, "Optical and magnetooptical tensor spectra of bismuth-substituted yttrium iron garnet films," J. Appl. Phys 68, 3544-3553 (1990).

22. K. Yasuhiro, K. Sato, K. Tamanoi, T. Saito, T. Tsushima, "Optical absorption spectra in cobalt-substituted epitaxial magnetic garnet films measured by photothermal deflection spectroscopy," Japanese Journal of Applied Physics 28, L656-L658 (1989).

23. V. J. Fratello and R. Wolfe, "Epitaxial garnet films for nonreciprocal magneto-optic devices," Handbook of Thin Film Devices 4: Magnetic Tin Film Devices, Chapter 3, Academic Press (2000).

24. A. Figotin and I. Vitebskiy, "Nonreciprocal magnetic photonic crystals," Phys. Rev. E 63, 066609 (2001).

25. H. Ramezani, S. Kalish, I. Vitebskiy, and T. Kottos "Unidirectional lasing emerging from frozen light in nonreciprocal cavities," Phys. Rev. Lett. 112, 043904 (2014). 
26. H. Nishihara, M. Haruna, T. Suhara "Optical Integrated Circuits" 


\section{APPENDIX}

Derivation of dispersion relation:

The electric and magnetic fields can be represented, generally in the planewave form, as

$\bar{E}=\overline{E_{o}}(x, y) \mathrm{e}^{i(\omega t-\beta z)}$

$\bar{H}=\overline{H_{o}}(x, y) e^{i(\omega t-\beta z)}$

Then the Maxwell equations, with the permittivity tensor represented as a matrix, become:

$\bar{\nabla} \times \bar{H}=\varepsilon_{o}\left(\begin{array}{ccc}\varepsilon_{c} & 0 & i g \\ 0 & \varepsilon_{c} & 0 \\ -i g & 0 & \varepsilon_{c}\end{array}\right) \frac{\partial \bar{E}}{\partial t}=\varepsilon_{o}\left(\begin{array}{ccc}\varepsilon_{c} & 0 & i g \\ 0 & \varepsilon_{c} & 0 \\ -i g & 0 & \varepsilon_{c}\end{array}\right) i \omega \bar{E}$

$\bar{\nabla} \times \bar{E}=-\mu_{o} \frac{\partial \bar{H}}{\partial t}=-\mu_{o} i \omega \bar{H}$

Where $\bar{\nabla}=\left(\begin{array}{ccc}\hat{x} & \hat{\mathrm{y}} & \hat{\mathrm{z}} \\ \frac{\partial}{\partial x} & \frac{\partial}{\partial y} & \frac{\partial}{\partial z} \\ H_{\mathrm{x}} & H_{\mathrm{y}} & H_{\mathrm{z}}\end{array}\right)$

Thus, on separating all the components, we get the following six equations:

$$
\begin{gathered}
\frac{\partial H_{\mathrm{z}}}{\partial \mathrm{y}}-\frac{\partial H_{y}}{\partial z}=i \omega \varepsilon_{o}\left(\varepsilon_{c} E_{x}+i g E_{z}\right) \\
\frac{\partial E_{z}}{\partial y}-\frac{\partial E_{y}}{\partial z}=-\mu_{o} i \omega H_{x} \\
-\frac{\partial H_{z}}{\partial x}+\frac{\partial H_{x}}{\partial z}=i \omega \varepsilon_{o} \varepsilon_{c} E_{y}
\end{gathered}
$$




$$
\begin{gathered}
-\frac{\partial E_{z}}{\partial x}+\frac{\partial E_{x}}{\partial z}=-\mu_{o} i \omega H_{y} \\
\frac{\partial H_{y}}{\partial x}-\frac{\partial H_{x}}{\partial y}=i \omega \varepsilon_{o}\left(-i g E_{x}+\varepsilon_{c} E_{z}\right) \\
\frac{\partial E_{y}}{\partial x}-\frac{\partial E_{x}}{\partial y}=-\mu_{o} i \omega H_{z}
\end{gathered}
$$

For a TM mode, which we study, $H_{z}=0$. This, combining with the above equations, leaves the only non-zero components as $H_{y}, E_{z}$ and $E_{x}$.

This leaves us with the following equations:

$$
\begin{gathered}
\frac{\partial H_{y}}{\partial z}=-i \omega \varepsilon_{o}\left(\varepsilon_{c} E_{x}+i g E_{z}\right) \\
-\frac{\partial E_{z}}{\partial x}+\frac{\partial E_{x}}{\partial z}=-\mu_{o} i \omega H_{y} \\
\frac{\partial H_{y}}{\partial x}=i \omega \varepsilon_{o}\left(\varepsilon_{c} E_{z}-i g E_{x}\right)
\end{gathered}
$$

Differentiating (C) w.r.t. $x$ gives

$$
\frac{\partial^{2} H_{y}}{\partial x^{2}}=i \omega \varepsilon_{o}\left(\varepsilon_{c} \frac{\partial E_{z}}{\partial x}-i g \frac{\partial E_{x}}{\partial x}\right)
$$

We note that $\frac{\partial}{\partial z}$ can be effectively replaced by $-i \beta$. Then

$\operatorname{From}(\mathrm{A}): \quad H_{y}=\frac{\omega \varepsilon_{o}}{\beta}\left(\varepsilon_{c} E_{x}+i g E_{z}\right)$

From (B): $\quad \frac{\partial E_{z}}{\partial x}=\mu_{o} i \omega H_{y}-i \beta E_{x}$

Differentiating (E) w.r.t. $x$ and comparing with $(\mathrm{C})$ gives

$$
\frac{\partial}{\partial x} E_{x}=i \beta E_{z}+\frac{g \mu_{o} \omega}{\varepsilon_{c}} H_{y}
$$


Substituting (F) and (G) into (D) gives

$$
\begin{aligned}
& \frac{\partial^{2}}{\partial x^{2}} H_{y}=i \omega \varepsilon_{o} \varepsilon_{c} \frac{\partial}{\partial x} E_{z}+g \omega \varepsilon_{o} \frac{\partial}{\partial x} E_{x} \\
& =i \omega \varepsilon_{o} \varepsilon_{c}\left(\mu_{o} i \omega H_{y}-i \beta E_{x}\right)+g \omega \varepsilon_{o}\left(i \beta E_{z}+\frac{g \mu_{o} \omega}{\varepsilon_{c}} H_{y}\right) \\
& =-\omega^{2} \varepsilon_{o} \mu_{o} \varepsilon_{c} H_{y}+\underbrace{\omega \beta \varepsilon_{o} \varepsilon_{c} E_{x}+i \beta g \omega \varepsilon_{o} E_{z}}_{\beta \omega \varepsilon_{o}\left(\varepsilon_{c} E_{x}+i_{g} E_{z}\right)=\beta^{2} H_{y}}+\frac{g^{2} \omega^{2}}{\varepsilon_{c}} H_{y} \\
& =\omega^{2} \mu_{o} \varepsilon_{o}\left(\frac{g^{2}}{\varepsilon_{c}}-\varepsilon_{c}\right) H_{y}+\beta^{2} H_{y}
\end{aligned}
$$

We define $k_{o}=\frac{\omega}{c}$, such that

$$
\begin{gathered}
\mu_{o} \varepsilon_{o} \omega^{2}=\frac{\omega^{2}}{c^{2}}=k_{o}^{2} \\
\Rightarrow \frac{\partial^{2}}{\partial x^{2}} H_{y}+\left[k_{o}^{2}\left(\varepsilon_{c}-\frac{g^{2}}{\varepsilon_{c}}\right)-\beta^{2}\right] H_{y}=0
\end{gathered}
$$

Defining $\varepsilon_{\text {eff }}=\left(\varepsilon_{c}-\frac{g^{2}}{\varepsilon_{c}}\right)$ as an effective permittivity in the cover, we get:

$$
\frac{\partial^{2}}{\partial x^{2}} H_{y}+\left(k_{o}^{2} \varepsilon_{e f f}-\beta^{2}\right) H_{y}=0
$$

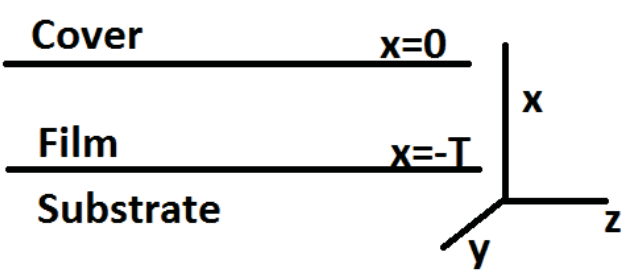

The Boundary conditions are that $E_{z}$ and $H_{y}$ are continuous across the layers. 
We define $\gamma_{\mathrm{eff}}, \mathrm{k}_{\mathrm{x}}$ and $\gamma_{\mathrm{s}}$ as the propagation constants in the cover, film and substrate, respectively. Then the boundary conditions give the results:

$$
\begin{aligned}
& H_{y}=H_{c} e^{-\gamma_{e f f} x}, x>0 \\
& H_{y}=H_{f} \cos \left(k_{x} x+\phi_{c}\right),-\mathrm{t}<\mathrm{x}<0 \\
& H_{y}=H_{s} \exp \left(\gamma_{s}(x+T)\right), x<-T
\end{aligned}
$$

Continuity of $H_{y}$ and $E_{z}$ at $x=0 \quad$ (Cover-Film Boundary) gives

Continuity of $H_{y}$ :

$$
H_{c}=H_{f} \cos \phi_{c}
$$

Continuity of $E_{z}$ :

From film side,

$$
\begin{aligned}
& H_{y}=\frac{\omega \varepsilon_{o}}{\beta} \varepsilon_{f} E_{x} \Rightarrow E_{x}=\frac{\beta}{\omega \varepsilon_{o} \varepsilon_{f}} H_{y} \\
& \frac{\partial H_{y}}{\partial x}=\frac{\omega \varepsilon_{o} \varepsilon_{f}}{\beta} \frac{\partial E_{x}}{\partial x}
\end{aligned}
$$

But since $g=0$ for the film, we also have

$$
\begin{aligned}
& \frac{\partial H_{y}}{\partial x}=i \omega \varepsilon_{o} \varepsilon_{f} E_{z} \\
\Rightarrow \quad & i \omega \varepsilon_{o} \varepsilon_{f} E_{z}=\frac{\omega \varepsilon_{o} \varepsilon_{f}}{\beta} \frac{\partial E_{y}}{\partial x} \\
& E_{z}=\frac{1}{i \beta} \frac{\partial E_{x}}{\partial x}=\frac{1}{i \beta} \frac{\beta}{\omega \varepsilon_{o} \varepsilon_{f}} \frac{\partial H_{y}}{\partial x}
\end{aligned}
$$




$$
\begin{aligned}
& =\frac{1}{i \omega \varepsilon_{o} \varepsilon_{f}} \frac{\partial}{\partial x} H_{y} \\
& =\frac{1}{i \omega \varepsilon_{o} \varepsilon_{f}} H_{f}\left[-\sin \left(u_{x} x+\phi_{c}\right) k_{x}\right]
\end{aligned}
$$

Thus, at $x=0$,

$$
E_{z, \mathrm{x}=0 \mid \text { film }}=\frac{-H_{f} \cdot \sin \phi_{c} k_{x}}{i \omega \varepsilon_{o} \varepsilon_{f}}=\frac{i H_{f} k_{x} \sin \phi_{c}}{\omega \varepsilon_{o} \varepsilon_{f}}
$$

On the cover side,

Eliminating $E_{x}$,

$$
\begin{aligned}
& g\left(H_{y}=\frac{\omega \varepsilon_{o} \varepsilon_{c}}{\beta} E_{x}+\frac{i g \omega \varepsilon_{o}}{\beta} E_{z}\right) \\
& \frac{-\frac{\varepsilon_{c}}{\beta}\left(\frac{\partial}{\partial x} H_{y}=i \omega \varepsilon_{o} \varepsilon_{c} E_{z}+g \omega \varepsilon_{o} E_{x}\right)}{g H_{y}-\frac{\varepsilon_{c}}{\beta} \frac{\partial}{\partial x} H_{y}=\frac{i \omega \varepsilon_{o}}{\beta}\left(g^{2}-\varepsilon_{c}^{2}\right) E_{z}} \\
& \Rightarrow E_{z|x=0| c o v e r}=\frac{g H_{c}+\frac{\varepsilon_{c}}{\beta} \gamma_{e f f} H_{c}}{\frac{i \omega \varepsilon_{o}}{\beta}\left(g^{2}-\varepsilon_{c}^{2}\right)}
\end{aligned}
$$

Equating with the value from the film side and substituting $H_{c}=H_{f} \cos \phi_{c}$ 


$$
\left(\frac{g+\frac{\varepsilon_{c}}{\beta} \gamma_{e f f}}{\frac{i \omega \varepsilon_{o}}{\beta}\left(g^{2}-\varepsilon_{c}^{2}\right)}\right) H_{f} \cos \phi_{c}=\frac{i H_{f} k_{x} \sin \phi_{c}}{\omega \varepsilon_{o} \varepsilon_{f}}
$$

gives

$$
\tan \phi_{c}=\frac{\varepsilon_{f}}{k_{x}}\left[\frac{g \beta+\varepsilon_{c} \gamma_{e f f}}{\varepsilon_{c}^{2}-g^{2}}\right]
$$

Continuity at the film-substrate boundary gives

Continuity of $H_{y}$ :

$$
H_{s}=H_{f} \cos \left(-k_{x} T+\phi_{c}\right)
$$

Continuity of $E_{z}$ :

Using the above and the expression $E_{z}=\frac{1}{i \omega \varepsilon_{o} \varepsilon} \frac{\partial H_{y}}{\partial x}$ obtained from Maxwell's equations, we get

$\frac{1}{i \omega \varepsilon_{o} \varepsilon_{f}}\left[-\sin \left(-k_{x} T+\phi_{c}\right) k_{x}\right] H_{f}=\frac{1}{i \omega \varepsilon_{o} \varepsilon_{s}} \gamma_{s} H_{s}=\frac{1}{i \omega \varepsilon_{o} \varepsilon_{s}} \gamma_{s} H_{f} \cos \left(-k_{x} T+\phi_{c}\right)$

Thus,

$$
\tan \left(k_{x} T-\phi_{c}\right)=\frac{\gamma_{s} \varepsilon_{f}}{k_{x} \varepsilon_{s}}
$$

Comparing (H) and (I) gives

$$
k_{x} T=\tan ^{-1}\left(\frac{\varepsilon_{f}}{k_{x}}\left[\frac{g \beta+\varepsilon_{c} \gamma_{\text {eff }}}{\varepsilon_{c}^{2}-g^{2}}\right]\right)+\tan ^{-1}\left[\left(\frac{\gamma_{s}}{k_{x}}\right)\left(\frac{\varepsilon_{f}}{\varepsilon_{s}}\right)\right]
$$




$$
=\tan ^{-1}\left\{\frac{\varepsilon_{f}}{k_{x}}\left[\frac{\gamma_{\text {eff }}}{\left(\varepsilon_{c}-\frac{g^{2}}{\varepsilon_{c}}\right)}+\frac{\beta_{g}}{\varepsilon_{c}\left(\varepsilon_{c}-\frac{g^{2}}{\varepsilon_{c}}\right)}\right]\right\}+\tan ^{-1}\left[\left(\frac{\varepsilon_{f}}{k_{x}}\right)\left(\frac{\gamma_{s}}{\varepsilon_{s}}\right)\right]
$$

Observing $\varepsilon_{e f f}=\varepsilon_{c}-\frac{g^{2}}{\varepsilon_{c}}$; we have the equation in the text. 